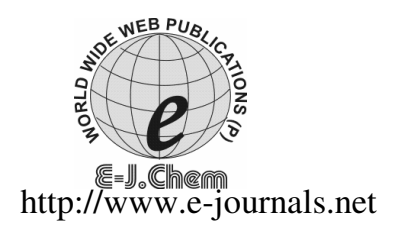

ISSN: 0973-4945; CODEN ECJHAO

E-Journal of Chemistry

2011, 8(3), 1086-1093

\title{
Methylene Blue Sensitized Photodechlorination of Isomeric Mono- and Dichloroanilines via Molecular Complex Formation Mechanism
}

\author{
U. C. PANDE and A. H. DWIVEDI* \\ Department of Chemistry, University School of Science \\ Gujarat University, Navrangpura, Ahmedabad-380 009, Gujarat, India \\ dwivedi_ank@indiatimes.com
}

Received 5 December 2010; Accepted 23 January 2011

\begin{abstract}
The photosensitized dechlorination of isomeric mono- and dichloroanilines has been studied using methylene blue as photosensitizer in alkaline medium. The dechlorination products have been identified and formation of molecular complex between aniline and methylene blue has been observed. The effects of the $\mathrm{pH}$, concentration of the sensitizer, concentration of the substrate, the intensity of the light and the temperature on the rate of the reaction have been studied. The quantum efficiency of the photodechlorination has been evaluated. The mechanism of the photodechlorination has been suggested.
\end{abstract}

Keywords: Photosensitized, Dechlorination, Chloroanilines, Dichloroanilines, Methylene blue

\section{Introduction}

Anilines and their derivatives are used in the manufacture of azo dyes, medicines, pesticides, polymers, resins, varnishes, perfumes, shoe blacks, vulcanizing rubber and as solvent. Aniline hydrochloride is used in pharmaceuticals and as an intermediate ${ }^{1-3}$. Chloroanilines and other aniline derivatives are used for the inhibition of copper metal corrosion in hydrochloric acid medium ${ }^{4}$. Chloroanilines are grouped as hazardous compounds and show toxicity by inhalation, ingestion or cutaneous absorption. The compounds show acute toxicity such as cyanosis, vertigo, headache and mental confusion. Chronic toxicity like anemia, anorexia, weight loss and cutaneous lesions are also reported ${ }^{5-7}$. Plenty of work is being carried out nowadays on aniline and its derivatives. A number of articles are published in the journals, magazines and books today all over the world explaining the different types of reactions of aniline and its compounds ${ }^{8-10}$. Amongst all aniline derivatives, haloderivatives have been reported as an environment pollutant in the literature ${ }^{11-12}$. Different techniques have been used to degrade these compounds. Several methods for biodegradation 
of chloroanilines (CA) and dichloroanilines (DCA) have been adopted ${ }^{13-15}$. Many articles have been published for the treatment of wastewater with ozone and different chemicals contaminated by haloanilines.

Amongst all these techniques, the photochemical degradation technique has been found very effective for the decomposition of chloroanilines especially by using UV-vis radiation in different solvents ${ }^{16-17}$. In our previous publications, we have reported the photosensitized dechlorination of isomeric mono- and dichloronitrobenzenes, isomeric chloroanisidines and isomeric mono- and dichlorobenzoic acids ${ }^{18-20}$. The present study has been undertaken for the photosensitized dechlorination of isomeric mono- and dichloroanilines in alkaline medium in visible light using $100 \mathrm{~W}$ tungsten lamp for the irradiation. Methylene blue (MB) has been used as photosensitizer, which has also been used as sensitizer in a number of photochemical reactions ${ }^{21-23}$.

The dechlorination products have been identified and the formation of a molecular complex between aniline and methylene blue has been observed. Several molecular complexes have been reported in literature ${ }^{24-26}$. The effect of the different parameters e.g. $\mathrm{pH}$, concentration of the sensitizer, concentration of the substrate, the intensity of the light and the temperature on the rate of the photodechlorination have been studied and reaction conditions have been established. The quantum efficiency of the photodechlorination has been evaluated using potassium ferrioxalate actinometer. The effect of the substrate concentration on quantum efficiency has been studied to evaluate the different excited state of the substrate molecule. The removal of chlorine from the substrate has been tested with silver nitrate. The mechanism of the photodechlorination has been suggested.

\section{Experimental}

All chemicals that have been used for the experiments are of AR grade (Aldrich) and no further purification has been done. Absolute alcohol (99\%) has been used after distillation. Double distilled water has been used for the dilution.

\section{Instrumentation}

$100 \mathrm{~W}$ tungsten filament light source (Phillips) has been used for the exposure of the sample solution. A convex lens has been used to converge the irradiation. A glass water jacket has been used to decrease the temperature of the solution. All the spectral measurements have been done on UV Vis spectrophotometer (Spectrascan-2800-Cibacorning, England). pH of the solution has been measured using $\mathrm{pH}$ meter (Systronics, India).

\section{Procedure}

Four sets of the experiment were prepared by pipetting $4 \mathrm{~mL}$ of $1.0 \times 10^{-3} \mathrm{M}$ solution of chloroanilines, $10 \mathrm{~mL}$ of $1.0 \times 10^{-1} \mathrm{M}$ solution of sodium hydroxide in each $50 \mathrm{~mL}$ volumetric flask and $2 \mathrm{~mL} 1.0 \times 10^{-4} \mathrm{M}$ solution of methylene blue was added to two flasks and solutions of all the four flasks were made up to the mark with double distilled water. The final concentrations of chloroanilines and methylene blue were $8.0 \times 10^{-5} \mathrm{M}$ and $4.0 \times 10^{-6} \mathrm{M}$ respectively. The measurements were carried out at $\mathrm{pH} 12$.

Two flasks, each containing methylene blue and without methylene blue were kept in the dark for $24 \mathrm{~h}$ while remaining similar flasks were exposed to visible light from $100 \mathrm{~W}$ tungsten lamp. The course of the reaction was followed by recording the spectrum of the exposed solution with a control solution in the range of $200-400 \mathrm{~nm}$ against reagent blank. The flask kept in the dark and flask exposed without sensitizer did not show difference in the 
spectrum when compared to the control; while the exposed flask containing sensitizer showed shift in $\lambda_{\max }$ from the original spectrum. This confirms that reaction takes place only in the presence of the methylene blue on irradiation. Thus, the reaction is photosensitized dechlorination and not normal or thermal dechlorination.

Free chloride was tested in the exposed solution with silver nitrate by observing white precipitate of $\mathrm{AgCl}$. This was compared with the control experiment run as blank without sample in which the $\mathrm{Cl}^{-}$test was found negative. The temperature of the solution was maintained at $298 \mathrm{~K}$ using water jacket. The quantum efficiency $(\phi)$ of the photodechlorination has been evaluated using potassium ferrioxalate actinometer.

\section{Molar absorptivities of the reactants and products}

The molar absorptivities of the substrates and the products have been calculated by measuring the absorbance of the solutions of known concentrations (Table 1). It has been observed that in the case of $p$-CA, the reaction goes to the completion but it is comparatively slower for $m$-CA and very slow and doesn't go to the completion in the case of $o$-CA. 2,6-DCA does not show any reaction on exposure.

\section{The reaction rate}

The increase in the absorbance of the reaction mixture at $310 \mathrm{~nm}$ has been measured by withdrawing $5 \mathrm{~mL}$ aliquot of the solution at different time intervals. The absorbance of the reaction mixture increased and became constant, indicating the completion of the reaction. The increase in the absorbance has been used to calculate the rate of the reaction. A plot of $2+\log (\mathrm{OD})$ (Optical Density) vs. time has been plotted and was found to be straight line with a positive slope. This indicates that the reaction follows first order reaction kinetics. The rate constant of the reaction has been determined by using the following expression:

\section{Results and Discussion}

$$
\text { Rate constant }=2.303 \times \text { slope }
$$

\section{Spectral characteristics}

The spectra of all chloro and dichloroanilines were recorded in the range of 200-400 nm under the experimental conditions against the reagent blank. The absorption bands at $232 \mathrm{~nm}$, $235 \mathrm{~nm}, 238 \mathrm{~nm}$ and $240 \mathrm{~nm}$ correspond to $\pi-\pi^{*}$ transition for $o$-CA, $m$-CA $p$-CA and 2,4-DCA respectively, which have higher molar absorptivity; while absorption bands at $283 \mathrm{~nm}, 285 \mathrm{~nm}, 289 \mathrm{~nm}$ and $295 \mathrm{~nm}$ correspond to non-bonding transition for $o$-CA, $m$-CA $p$-CA and 2,4-DCA respectively, which have lower molar absorptivities (Table 1). The spectra of unexposed solutions of aniline and chloroanilines containing MB were recorded and it was observed that the spectra matched with the original samples.

The spectra of exposed solutions of $o$-CA, $m$-CA, $p$-CA and 2,4-DCA were recorded after withdrawing aliquots at different time intervals. The $\pi-\pi^{*}$ bands changed to $230 \mathrm{~nm}$ and $\mathrm{n}-\pi^{*}$ bands were shifted to $310 \mathrm{~nm}$ for all the compounds. The molar absorptivity of non-bonding transition band also increased (Table 1). The spectrum of aniline has also been recorded under the similar experimental conditions to study the photochemical behavior of aniline, which showed $\lambda_{\max }$ at $230 \mathrm{~nm}$ and $280 \mathrm{~nm}$. The spectrum of the exposed solution of aniline showed $\lambda_{\max }$ at $230 \mathrm{~nm}$ and $310 \mathrm{~nm}$. The spectra of the chloroanilines after exposure show the similar characteristics of $\lambda_{\max }$ and absorptivity as of the pure aniline. Therefore it has been concluded that all chloro- and dichloroanilines are converted into aniline after dechlorination. 2,6-DCA does not undergo decomposition under the experimental conditions and does not form molecular complex. $o$-CA molecule shows extremely slow reaction. 
Table 1. Experimental values of $\lambda_{\max }$ and molar absorptivity $(\varepsilon)$ of aniline and chloroanilines

\begin{tabular}{ccc}
\hline \multirow{2}{*}{ Compound } & $\begin{array}{c}\text { Experimental } \\
\lambda_{\max }(\text { (qvalue })\end{array}$ & $\begin{array}{c}\text { Product } \\
\lambda_{\max }(\text { \&value })\end{array}$ \\
\hline \multirow{2}{*}{${\mathrm{Ph}-\mathrm{NH}_{2}}^{230(8490)}$} & $230(8490)$ \\
$o-\mathrm{CA}$ & $280(1420)$ & $310(4090)$ \\
& $232(7790)$ & $230(8100)$ \\
$m-\mathrm{CA}$ & $283(2200)$ & $310(3540)$ \\
& $235(9240)$ & $230(8760)$ \\
$p-\mathrm{CA}$ & $285(1980)$ & $310(3808)$ \\
& $238(11080)$ & $230(8530)$ \\
$2,4-\mathrm{DCA}$ & $289(1310)$ & $310(4000)$ \\
& $240(9940)$ & $230(8990)$ \\
$2,6-\mathrm{DCA}$ & $295(2080)$ & $310(3928)$ \\
& $210(18910)$ & - \\
\hline
\end{tabular}

\section{The rate of the reaction}

The increase in the absorbance at $310 \mathrm{~nm}$ has been measured for isomeric chloroanilines at different time intervals; which increased and became constant indicating the completion of the reaction. The increase in the product concentration increases the absorbance at $310 \mathrm{~nm}$, which has been used to calculate the rate constant. The rates of the dechlorination for $o-, m-, p$-chloroanilines and 2,4-dichloroaniline have been calculated (Table 2). The photosensitized dechlorination shows different rates for different mono- and dichloroanilines in experimental conditions (Table 2). The order of the dechlorination follows $p$ - $>m$ - $>o$-chloroaniline. However 2,6-DCA does not undergo dechlorination reaction. The 2,4 -DCA shows a reaction rate that is higher than $m$-CA but lower than $p$-CA. The experimental results show that both the chlorine atoms are removed simultaneously.

Table 2. Rates of photosensitized dechlorination of chloroanilines

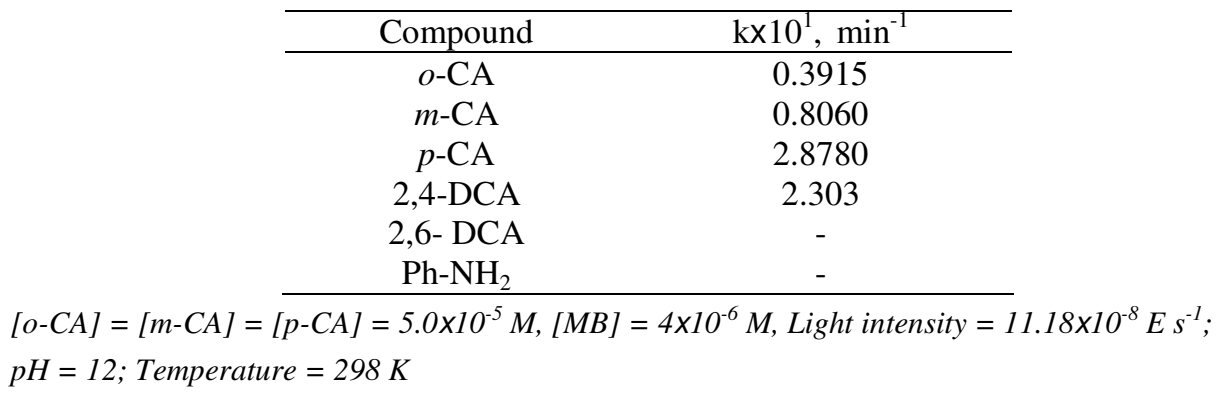

The effect of the variables on the rate of the photodechlorination of chloroanilines Effect of the $\mathrm{pH}$

Methylene blue doesn't work as a photosensitizer in the acidic and neutral medium for this reaction. The rate constant of the photosensitized reaction has been calculated at different $\mathrm{pH}$ for $o-, m$ - and $p$-chloroanilines between $8-13 \mathrm{pH}$. It has been observed that as $\mathrm{pH}$ increases, the rate of the reaction increases slowly, but between 11 to $12 \mathrm{pH}$, showed a large increase in the rate of the reaction has been observed and then it became constant. Therefore the $\mathrm{pH} 12$ has been selected for the subsequent studies. 


\section{Effect of the concentration of the sensitizer}

The effect of the different concentrations of methylene blue on the rate of the dechlorination has been studied by calculating the rate constants for $o-, m$ - and $p$-chloroanilines using the different concentrations of methylene blue in the range of $0.4 \times 10^{-5}-2.0 \times 10^{-5} \mathrm{M}$. The increase in the concentration of the sensitizer does not show change in the rate of the reaction. However the rate slightly decreases at the higher concentration of the sensitizer. It appears that the self-deactivation of the sensitizer takes place at higher concentration.

\section{Effect of the concentration of the substrate}

The effect of the different initial concentrations of $o-, m$ - and $p$-chloroanilines on the rate of the reaction has been studied in the range of $0.4 \times 10^{-4}-1.2 \times 10^{-4} \mathrm{M}$. The rate of the reaction remains constant with increase in the initial concentration of the substrate. This shows that the reaction is independent of the initial concentration of the substrate. However the rate of the dechlorination of $p$-CA is highest and $o$-CA is lowest.

\section{Effect of the light intensity}

The increase of the light intensity [Einstein / second (E/s)] shows positive effect and the rate of the dechlorination increases as the light intensity increases. The number of the excited molecules of the sensitizer increases with higher light intensity as the number of photons increases and corresponding the rate of the reaction increases (Table 3). A linear relationship is observed between the light intensity and the rate of the reaction.

Table 3. Effect of the light intensity

\begin{tabular}{cccc}
\hline $\begin{array}{c}\text { Light intensity, } \\
\mathrm{Ix} 10^{8}, \mathrm{E} \mathrm{sec}^{-1}\end{array}$ & $\begin{array}{c}\mathrm{kx} 10^{1}, \mathrm{~min}^{-1} \\
o-\mathrm{CA}\end{array}$ & $\begin{array}{c}\mathrm{kx} 10^{1}, \mathrm{~min}^{-1} \\
m \text {-CA }\end{array}$ & $\begin{array}{c}\mathrm{kx} 10^{1}, \mathrm{~min}^{-1} \\
p \text {-CA }\end{array}$ \\
\hline 4.47 & 0.1948 & 0.3768 & 1.3810 \\
6.72 & 0.2405 & 0.4915 & 1.8420 \\
11.18 & 0.3838 & 0.8060 & 2.8180 \\
\hline
\end{tabular}

$[o-C A]=[m-C A]=[p-C A]=5.0 \times 10^{-5} M,[M B]=4 \times 10^{-6} M, p H=12 ;$ Temperature $=298 \mathrm{~K}$

Table 4. Quantum efficiencies of chloroanilines

\begin{tabular}{cccccc}
\hline Compound & $o$-CA & $m$-CA & $p$-CA & $2,4-\mathrm{DCA}$ & $2,6-\mathrm{DCA}$ \\
$\Phi$-Value & 0.4041 & 1.0377 & 1.1579 & 0.9831 & - \\
\hline
\end{tabular}

\section{Effect of the temperature}

The effect of the variation of the temperature has been studied between $298-328 \mathrm{~K}$ on the rate of the reaction. The rate of the reaction remains constant with the increase in the temperature in the above range. This indicates that the rate of the reaction is independent of the temperature and thermal condition does not affect the photodechlorination. The temperature independent nature of the rate of the reaction also indicates that the product formation takes place directly from the excited species without any intermediate stage.

\section{$\phi$ - Value}

The quantum efficiency has been calculated by using standard potassium ferrioxalate actinometer. $\phi$ values have been also calculated for the chloroaniline at different initial concentrations of the substrate (Table 4). There is a linear relationship between the initial concentration of the substrate and $\phi v a l u e$, suggesting that the $\phi$ values are dependent on the initial concentration of the substrate. The plot of the inverse of the quantum efficiency versus the inverse of the initial concentration of the substrate has been found to be linear with a positive slope which suggests that there is a direct product formation from the singlet excited state and not via triplet intermediate state ${ }^{27}$. 


\section{Molecular complex formation}

The spectrum of the exposed solution of aniline and isomeric chloroanilines compare well with each other. The formation of anilines from chloroanilines takes place. The band at $280 \mathrm{~nm}$ in aniline corresponds to $\mathrm{n}-\pi^{*}$ transition and shows lower absorptivity (Table 1 ). The exposed solution of aniline shows that this band is shifted to longer wavelength $310 \mathrm{~nm}$ with higher molar absorptivity. The formation of a molecular complex between excited aniline molecules with MB molecule has been suggested. There is no absorption band change in ground state. However excited chloroaniline molecule does not form a complex with MB. The excited molecule of chloroaniline undergoes two simultaneous processes viz. dechlorination and donation of nitrogen lone pair to MB molecule. The molecular complex thus formed exists in ground state. The molecular complex formation between aniline and $\mathrm{MB}$ on exposure takes place very fast while the formation of a molecular complex in the case of chloroanilines takes longer time, suggesting that complex formation takes place only after the dechlorination. The exposure of the isomeric chloroanilines with $\mathrm{MB}$ in alkaline medium excites the chloroaniline molecules to singlet-excited state by energy transfer from MB molecule. The presence of chlorine does not allow the formation of a molecular complex. The excited molecule of chloroaniline undergoes first dechlorination and then forms molecular complex. The rate of complex formation is proportional to the rate of dechlorination and thus the formation of aniline takes place. The absorption spectrum of MB was recorded before and after the exposure taking aniline as a blank, which did not show any change before and after exposure. The band at $280 \mathrm{~nm}$ of aniline gets shifted to $310 \mathrm{~nm}$ of aniline. Thus it can be concluded that aniline molecule only gets perturbed and forms charge transfer complex with MB.

\section{Mechanism}

Aniline and chloroanilines have their $\lambda_{\max }$ below $300 \mathrm{~nm}$. These molecules do not absorb the visible light and they are not photo-degraded directly. Methylene blue (MB) has $\lambda_{\max }$ at $665 \mathrm{~nm}$; which absorbs visible radiation and forms excited molecule. The absorption of the radiation at this $\lambda_{\max }$ results in the singlet excited state of methylene blue ${ }^{23}$. The energy transfer from singlet excited state of $\mathrm{MB}$ to aniline and isomeric chloroanilines takes place, resulting in the ground state of MB and singlet excited state of chloroanilines and aniline. The change in the spectrum of aniline has been observed after exposure along with MB. The absorption band of $\pi-\pi^{*}$ of aniline remains unchanged, but $\mathrm{n}-\pi_{*}$ bands shows red shift and with increased absorptivity ${ }^{28-31}$. It can be concluded that non-bonding electrons of aniline are excited in the lower vacant orbital of MB forming a molecular complex. The molecular complex shows higher absorptivity ${ }^{28-31}$ and longer wavelength absorption at $310 \mathrm{~nm}$ (Scheme 1). Energy transfer takes place from singlet-excited state of $\mathrm{MB}$ to isomeric chloroanilines. The excited chloro molecule undergoes $\mathrm{C}-\mathrm{Cl}$ bond cleavage forming $\mathrm{Cl}^{-}$ion. The excited chloroaniline molecule simultaneously undergoes dechlorination and transfer of non-bonding electrons of nitrogen atom to MB molecule, forming a molecular complex ${ }^{26,32}$. The molecular complex is in the ground state. The absorbance of the molecular complex remains constant with time. The spectral characteristics of dechlorinated chloroanilines are similar to the exposed aniline (Scheme 2). The new absorption band at $310 \mathrm{~nm}$ is due to transfer of non-bonding electrons of nitrogen atom of aniline to MB. The plot of the inverse of the quantum efficiency versus the inverse of the initial concentration of the substrate is linear with a positive slope; which indicates that there is a direct product formation from the singlet-excited state of the substrate and not via intermediate triplet $\operatorname{state}^{27}$. 


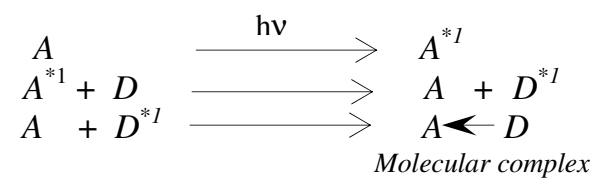

\section{Scheme 1}

MB

$$
\stackrel{h v}{\longrightarrow} \mathrm{MB}^{* 1}
$$

Methylene Blue

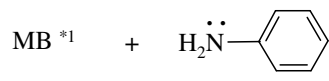

Aniline

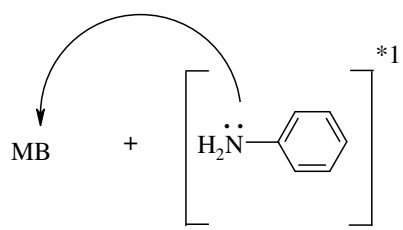

$\mathrm{MB}{ }^{* 1}$

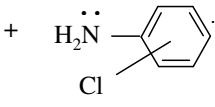

Chloroaniline
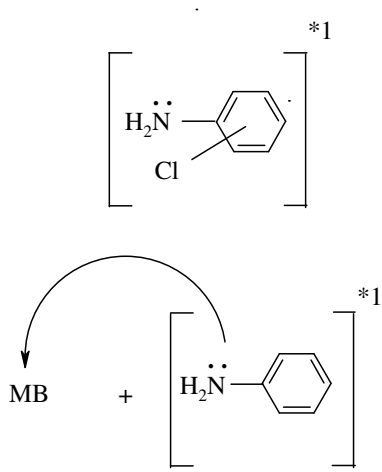

$\mathrm{Cl}+\mathrm{e}^{-}$
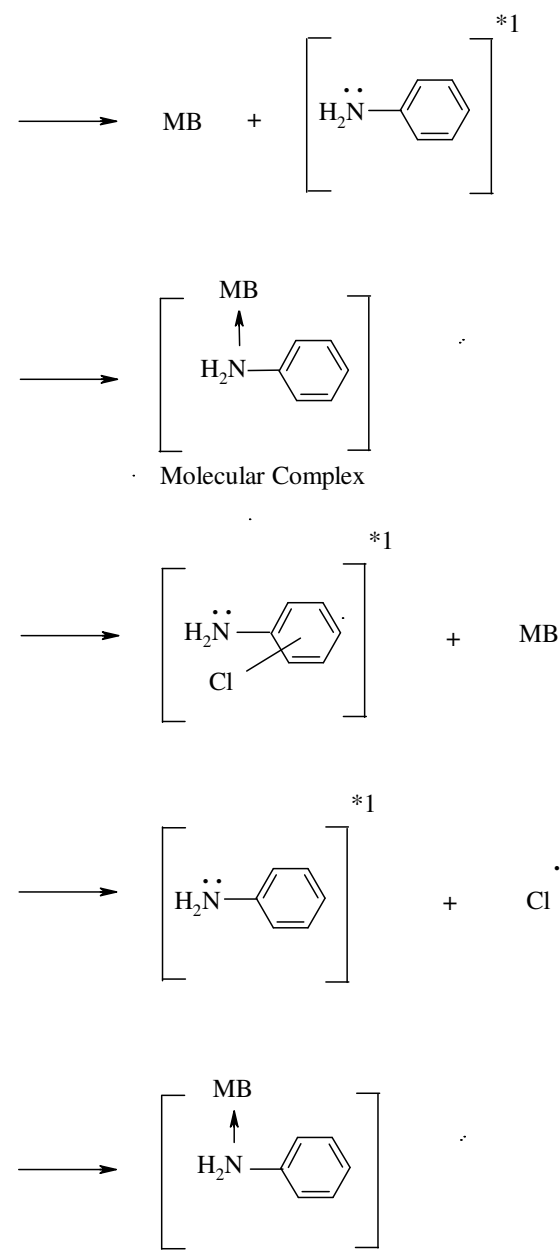

$\mathrm{Cl}$

Scheme 2

\section{Conclusion}

Isomeric chloro- and dichloroanilines undergo photosensitized dechlorination in the presence of methylene blue and visible light in alkaline medium. The spectral profiles of the exposed solution suggest that the product of the reactions is only aniline. The molecular complex 
between excited state of aniline and ground state of MB is formed. The study of different parameters concludes that the rate of the dechlorination is dependent on light intensity and $\mathrm{pH}$ and is independent of temperature, initial concentration of substrate and concentration of sensitizer. $\phi$ value is dependent on initial concentration of substrate. The rate of reaction follows the order $p$-CA $>m$-CA $>o$-CA.

\section{References}

1. The Merck Index, Version 12.3, Merck \& Co. Inc., NJ, USA, 1999.

2. Jen J-F, Chang C-T and Yang T C, J Chromatogr A, 2001, 930(1-2), 119-125.

3. Sarasa J, Roche M P, Ormad M P, Gimeno E, Puig A and Ovelleiro J L, Water Res., 1998, 32, 2721.

4. Khaled K F and Hackerman N, Electrochim Acta, 2004, 49, 485.

5. Gosselin R E, Hodge H C, Smith R P and Gleason M N, Clinical Toxicology of Commercial Products, $4^{\text {th }}$ Eds., (Williams and Wilkins, Baltimore), Section III, 1976, 29.

6. Adema D M M and Vink G J, Chemosphere, 1981, 10, 533-554.

7. Crossland N O, Chemosphere, 1990, 21, 1489-1497.

8. Chesta C A, Cosa J J and Previtali C M, J Photochem., 1987, 39, 251.

9. Soltermann A T, Chesta C A, Cosa J J and Previtali C M, An Asoc Quim Argent., 1989, 77(6), 487-498.

10. Szczepanik B and Latowski T, Pol J Chem., 1997, 71, 807.

11. Raymond J W, Rogers T N, Shonnard D R and Kline A A, J Haz Mat., 2001, 84, 189-215.

12. Barr D B and Needham L L, J Chromatography B, Anal Technol Biomed Life Sci., 2002, 778, 5.

13. Hwang H-M, McCullum D and Slaughter L, Bull Environ Contam Toxicol., 1998, 60, 81-87.

14. Susarla S, Masunaga S and Yonezawa Y, Microb Ecol., 1997, 33(3), 252-256.

15. Susarla S, Masunaga S and Yonezawa Y, Wat Sci Technol., 1996, 34, 489.

16. Hwang H-M, Hodson R E and Lee R F, Appl Environ Microbiol., 1986, 50, 1177-1180.

17. Ishikawa S, Baba K, Hanada Y, Uchimura Y and Kido K, Bull Environ Contam Toxicol., 1989, 42, 65.

18. Dwivedi A H and Pande U C, J Photochem Photobiol A Chem., 2003, 154, 303-309.

19. Dwivedi A H and Pande U C, J Indian Chem Soc., 2005, 82, 424-427.

20. Dwivedi A H and Pande U C, E-J Chem., 2010, 7(S1), S111- S120.

21. Jockusch S, Timpe H J, Schnabel W and Turro N J, J Phys Chem A, 1997, 101, 440-445.

22. Kojima M, Takagi T, Matsubara C and Nakamura K, Chem Lett., 2000, 4, 354-355.

23. Kojima M, Takagi T, Matsubara C and Nakamura K, J Photochem Photobiol A Chem., 2001, 74(3), 369-377.

24. Enescu M and Lindqvist L, J Phys Chem B, 1995, 99, 8405.

25. Enescu M, Ridard J, Gheorghe V and Levy B, J Phys Chem B, 2002, 106, 176-184.

26. Enescu M, Ridard J, Gheorghe V and Levy B, J Phys Chem B, 2000, 104, 1073-1077.

27. Freeman P K, Jang J S and Ramnath N, J Org Chem., 1991, 56, 6072.

28. Kross R D and Fassel V A, J Am Chem Soc., 1957, 79, 38.

29. Issa R M and El-Essawey M M, Z Phys Chem., (Leipzig), 1973, 253, 96.

30. Hindawey A M, Nassar A M G and Issa R M, Acta Chim., 1976, 88, 341.

31. Hindawey A M, Nassar A M G and Issa R M, Acta Chim., 1977, 92, 263.

32. Issa Y M, El-Kholy A E, Hindawey A M and Issa R M, J Indian Chem Soc., 1980, LVII, 216. 


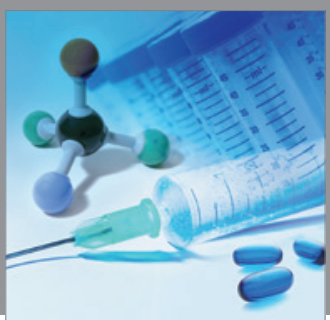

International Journal of

Medicinal Chemistry

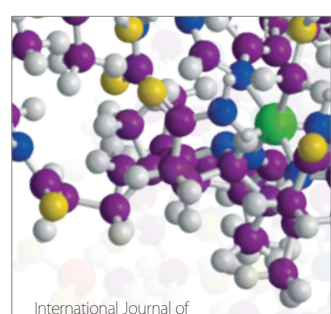

Carbohydrate Chemistry

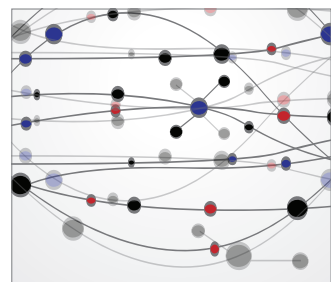

The Scientific World Journal
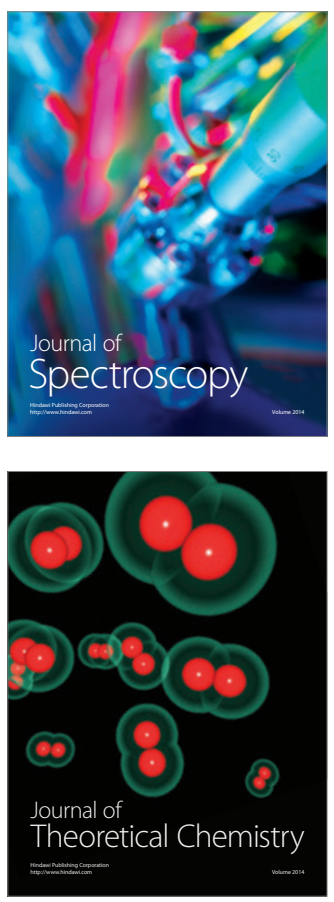
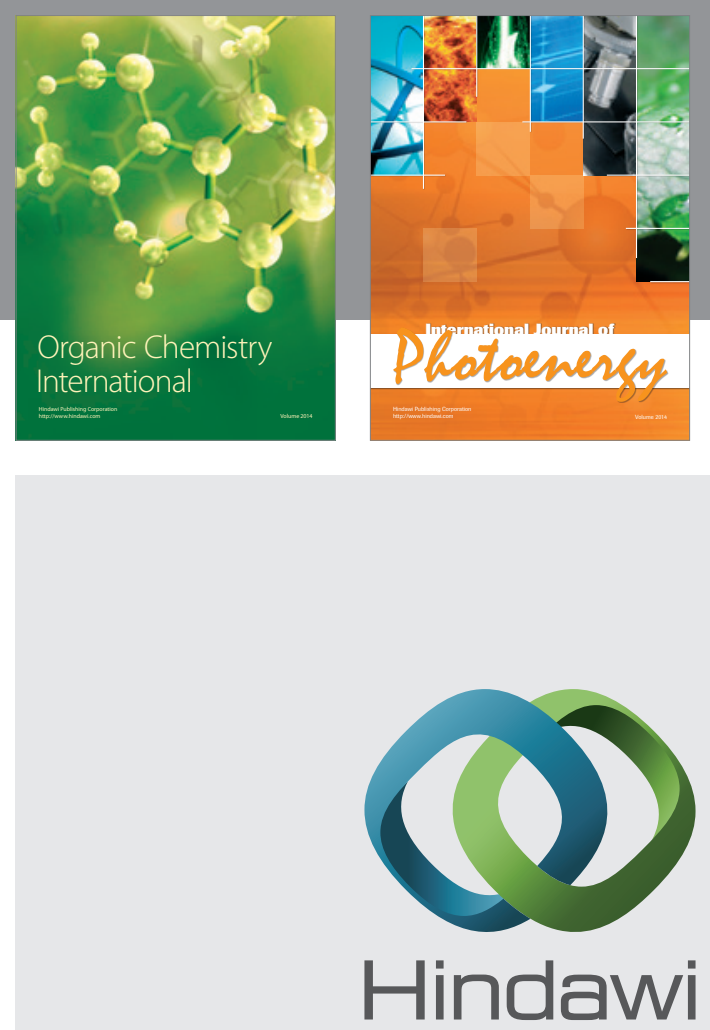

Submit your manuscripts at

http://www.hindawi.com
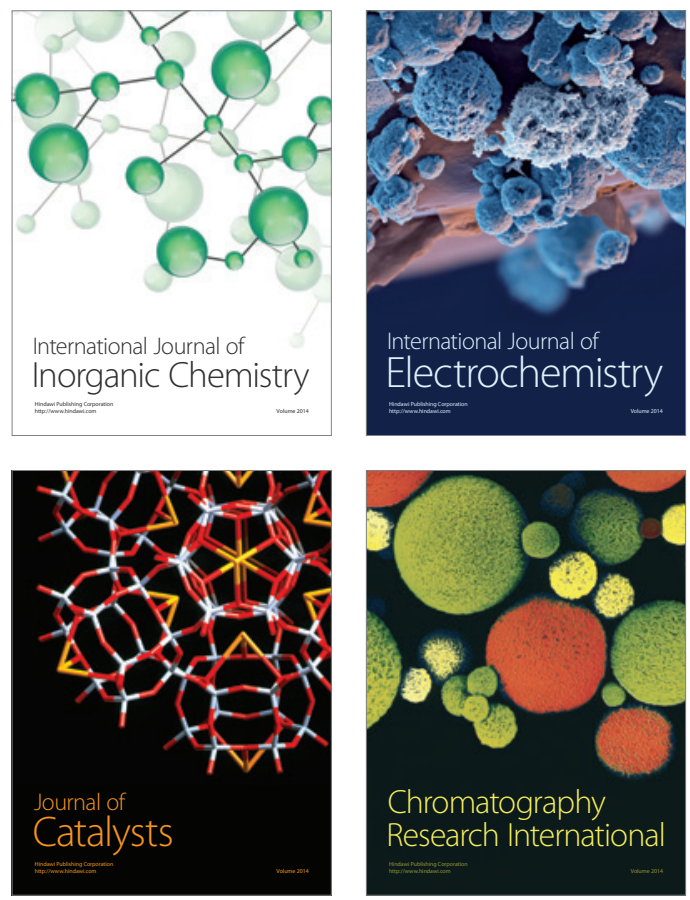
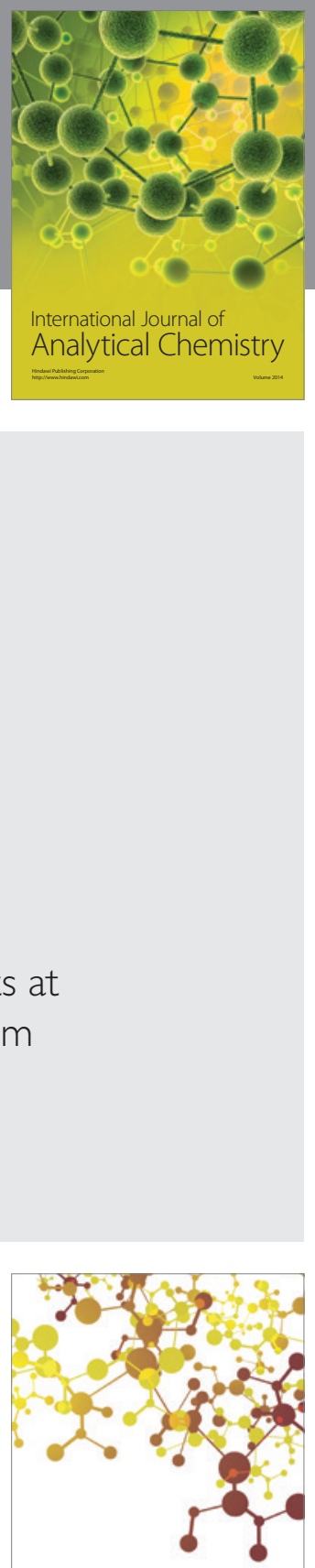

Journal of

Applied Chemistry
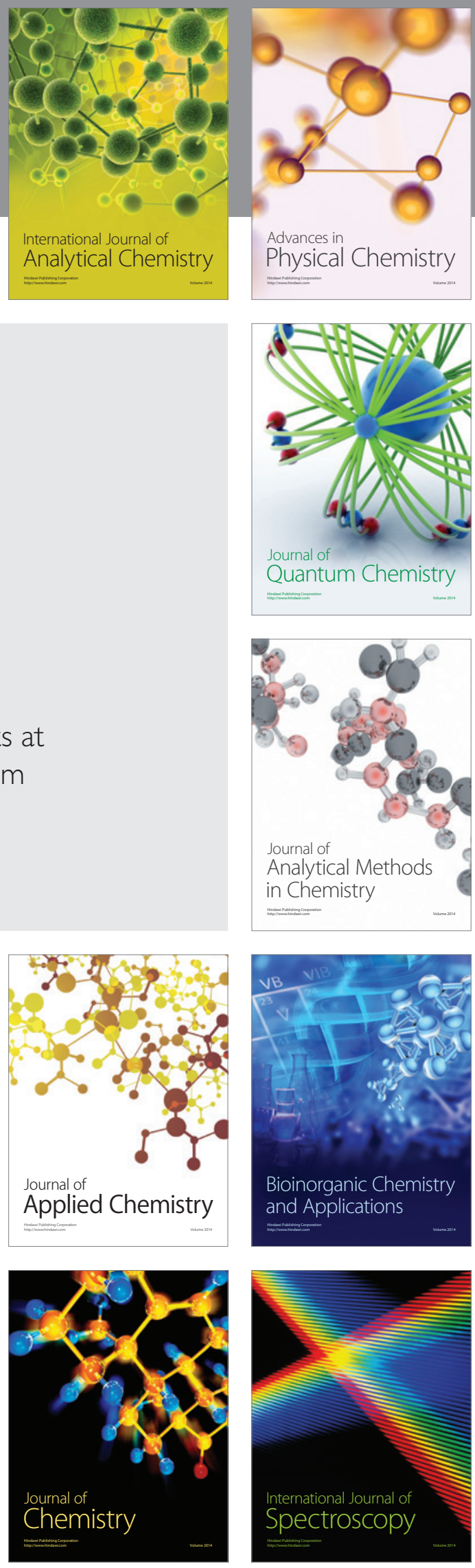